\title{
Studie 1: Zusammenhänge von Berufsorientierungsmaßnahmen und persönlichen Merkmalen Jugendlicher als Determinanten individualisierter schulischer Berufsorientierung
}

\subsection{Einleitung}

Die schulische Unterstützung beruflicher Orientierung, kurz BO, erfährt als ein wichtiger Faktor im Entscheidungsprozess zur Ausbildungs- und Studienwahl Jugendlicher seit mehreren Jahren eine hohe Aufmerksamkeit durch die Berliner Politik, Wirtschaft und das Schulwesen. Die gemeinsamen Bemühungen werden zum Beispiel an dem 2015 verabschiedeten Landeskonzept Berufs- und Studienorientierung Berlin, welches die BO im Curriculum aller weiterführenden Schulen stärkt beziehungsweise neu verankert, sichtbar (Berliner Senat, 2015). Die schulische BO dient der Begleitung des Berufswahlprozesses und der Entwicklungsförderung „berufswahlrelevanter Kompetenzen“ (Lipowski et al., 2015b, S. 14; vgl. auch Famulla, 2005; Hany \& Driesel-Lange, 2006).

Unter Berücksichtigung schulform- und -standortabhängiger Umsetzungsformate (vgl. Dreer, 2013b) definieren wir in dieser Arbeit schulische BO als die Einheit aller Maßnahmen der beruflichen Orientierung, die innerhalb der Unterrichtszeit durch Schulpersonal oder Kooperationspartner und Kooperationspartnerinnen angeleitet beziehungsweise durchgeführt werden. Ferner mit inbegriffen sind Angebote, an denen Schüler*innen auf Initiative der Schule, jedoch außerhalb der regulären Unterrichtszeit teilnehmen. Mehrere Herausforderungen, die in Berlin im bundesweiten Vergleich teilweise besonders konzentriert

Diese empirische Studie wurde 2017 in ähnlicher Form veröffentlicht und kann wie folgt zitiert werden: Ohlemann, S. \& Ittel, A. (2017). Zusammenhänge von Berufsund Studienorientierungsmaßnahmen und persönlichen Merkmalen von Jugendlichen als Determinanten individualisierter schulischer Berufs- und Studienorientierung? In T. Brüggemann, K. Driesel-Lange \& C. Weyer (Hrsg.), Instrumente der Berufsorientierung (S. 125-151). Münster: Waxmann. 
aufzufinden sind, erklären das gestiegene Interesse und Engagement der genannten Akteure: $11 \%^{1}$ der Berliner Jugendlichen beendeten 2008 ihre Schulzeit ohne Schulabschluss (Klemm, 2010, S. 12), $7 \%^{2}$ der Suchenden blieben im gleichen Jahr ohne Ausbildungsvertrag (Bundesagentur für Arbeit, 2015). Zudem erfordert die überaus heterogene Schülerschaft Berlins mit einem hohen Anteil an Lernenden nichtdeutscher Herkunftssprache $(37 \%)$ beziehungsweise aus sozial benachteiligten Familien $(34 \%)^{3}$ (Senatsverwaltung für Bildung, Jugend und Wissenschaft Berlin, 2015, S. H3) einen hohen Grad an Individualisierung der schulischen BO. Schließlich soll sie den unterschiedlichen Entwicklungsständen und Bedarfen der Lernenden gerecht werden und den unter anderem anhand der PISA-Studien empirisch nachgewiesenen Nachteilen in den Bildungskarrieren dieser beiden Gruppen entgegenwirken (vgl. OECD, 2014). Denn persönliche Merkmale, wie das Geschlecht, die Muttersprache oder die Schulleistung nehmen Einfluss auf die „Berufsorientierungs- und Übergangsprozesse“ der Lernenden (Rahn et al., 2014, S. 4; vgl. auch Tillmann, Schaub, Lex, Kuhnke \& Gaupp, 2014). Mehrere theoretische Modelle, wie das Thüringer Berufsorientierungsmodell (Driesel-Lange et al., 2010) oder das von Neuenschwander und Hartmann (2011) weiterentwickelte Phasenmodell zu Berufswahlprozessen (vgl. dazu auch Herzog et al., 2006) übertragen die Annahme individueller Entwicklungsverläufe bereits in Phasenmodelle der Berufswahl. Dennoch ist noch nicht geklärt, wie eine Bedarfsanalyse zur Entwicklung einer individualisierten BO stattfinden kann und wie die Ergebnisse in entsprechende BO-Maßnahmen fließen können, um Jugendliche nach ihrem persönlichen Entwicklungsstand, ihren Fähigkeiten und ihren Bedürfnissen zu fördern. In diesem Beitrag soll begonnen werden, dieser Frage auf den Grund zu gehen, indem aufgezeigt wird, in welchem Verhältnis persönliche Merkmale von Heranwachsenden mit dem persönlichen Nutzen von schulischen BO-Maßnahmen im Hinblick auf den subjektiven Stand der Berufswahlvorbereitung stehen.

\subsection{Forschungsstand}

Theoretischer Ausgangspunkt der hier vorliegenden Analyse ist der Zusammenhang zwischen Schulleistung, Geschlecht und Muttersprache Heranwachsender

\footnotetext{
${ }^{1}$ Bundesdurchschnitt: $8 \%$ (Klemm, 2010, S. 12)

${ }^{2}$ Bundesdurchschnitt: $3 \%$ (Bundesagentur für Arbeit, 2015)

${ }^{3}$ Schüler*innen mit Lernmittelbefreiung
} 
und ihrem Berufswahlverhalten. Der aktuelle Forschungsstand wird nachfolgend dargestellt.

\section{Die Rolle des Geschlechts in der Berufsorientierung}

Geschlechtsspezifische Varianzen im initialen Interesse an der Berufsfindung, im Berufswahlprozess und in der (ersten) Berufswahlentscheidung zeigten sich in mehreren nationalen und internationalen Studien (vgl. zsf. Sikora \& Pokropek, 2012). Jungen (Mädchen) weisen höhere Interessenswerte für Berufe, die mit naturwissenschaftlich-technischen (literarisch-sprachlich-künstlerischen) Unterrichtsfächern assoziiert werden, auf (Driesel-Lange, 2011) und interessieren sich eher für typisch männliche (weibliche) Berufe (Sinclair \& Carlsson, 2013). Schüler und Schülerinnen unterscheiden sich zudem in ihrem Berufswahlverhalten (Fend, 1991) und der Entwicklung einzelner Facetten ihrer Berufswahlreife (Cassie \& Chen, 2012): Mädchen recherchieren intensiver in der Vorbereitung auf die Berufswahl und fühlen sich dabei in fast doppelt so vielen Fällen als Jungen von der Entscheidungsaufgabe emotional negativ belastet (Fend, 1991). Eine Ursache geschlechtsspezifischer Unterschiede in der Berufswahl liegt in dem unterschiedlichen Rollenverständnis von Mädchen und Jungen (Schmude, 2010), das auch zu einem ungleichen Leistungswillen führen kann (Hurrelmann, 2009). Ein traditionelles Rollenverständnis führt bei vielen Jungen zu der Auffassung, dass ihnen ,ein beruflicher Erfolg per Tradition“ (Hurrelmann, 2009, S. 19) zustünde und hängt mit einer geringeren Leistungsmotivation im Beruf zusammen. Gemäß dem Stereotype Threat (Steele \& Aronson, 1995) benennen Sinclair und Carlsson (2013) die Präferenz für geschlechtsstereotypische Berufe als eine Konsequenz einer als hoch empfundenen Bedrohung der eigenen Geschlechtsidentität. Obwohl die beschriebenen empirischen Studien Geschlechterunterschiede im Berufswahlprozess zeigen, lassen sich ,stabile Effekte von Maßnahmen [zu geschlechtsunabhängiger Berufsorientierung] praktisch nicht nachweisen“ (Driesel-Lange, 2011, S. 218), sodass weitere Analysen notwendig sind.

\section{Schulische Leistungen als Faktor in der Berufsorientierung}

Auch die Schulleistung als Faktor in der Ausprägung der Berufsorientierung wurde bereits aus mehreren Perspektiven beleuchtet. Jugendlichen mit schlechten Schulnoten fällt zum Beispiel die Ergreifung ,rationale[r] Berufsfindungsprozesse“ im Abgangsjahr der Realschule eher schwer (Fend, 1991, S. 89). Ein schlechterer Notendurchschnitt beziehungsweise geringerer Schulabschluss wirkt sich darüber hinaus auch auf die Art der Ausbildungs- und Berufswahl aus. Jungen mit einem schlechteren Notendurchschnitt beziehungsweise geringerem Abschluss tendieren 
in ihrer Berufswahl zu Berufen mit hoher maskuliner Geschlechtstypik und münden eher in eine betriebliche Ausbildung (vgl. Busch, 2013; Kroll \& Granato, 2013; Schmude, 2010). Dagegen entwickeln Mädchen mit geringer traditioneller Geschlechtsrollenorientierung neutrale oder maskulin-besetzte Berufswünsche, je besser ihre schulischen Leistungen sind (vgl. Busch, 2013; Schmude, 2010). Die folgende Analyse widmet sich den noch nicht betrachteten Effekten von BO-Maßnahmen in Abhängigkeit verschiedener Leistungsprofile.

\section{Die Muttersprache als Einflussfaktor in der Berufsorientierung}

Die Muttersprache und der Migrationshintergrund sind, so indizieren es mehrere Studien, relevante Faktoren in allen Phasen der Berufsorientierung: Fends Studienergebnisse von 1991 legen nahe, dass junge Migrant*innen oft größere Schwierigkeiten als ihre Peers ohne Migrationshintergrund bei der Entscheidungsfindung für einen Beruf haben, obwohl ihre Familien über einen ähnlichen Leistungs- und Aufstiegswillen wie Familien ohne Migrationshintergrund und eine „ausgeprägte Bildungsorientierung“ (Beicht \& Granato, 2011, S. 20) verfügen. Diese hohe Erwartungshaltung hinsichtlich der Schul- und Berufsbildung richtet sich in gleichem Maße an beide Geschlechter (Boos-Nünning \& Karakaşoğl, 2004). Auch bezüglich der Intensität und des Berufsorientierungsverhaltens unterscheiden sich Jugendliche mit und ohne Migrationshintergrund nicht ausreichend, um eine hinreichende Erklärung zu liefern (vgl. zfs. Diehl, Friedrich \& Hall, 2009; Gaupp, Lex \& Reißig, 2011; Granato, 2013; Wippermann \& Flaig, 2009). Unklar ist auch, warum trotz einer ähnlichen Berufsorientierung Schüler*innen mit Migrationshintergrund in Deutschland weniger oft ein Studium aufnehmen als Schüler*innen ohne Migrationshintergrund (Lörz et al., 2012). Für die abweichenden beruflichen Bildungspläne von Migrant*innen ist also ein Zusammenspiel mit weiteren Ursachen beziehungsweise Faktoren, wie etwa den schulischen Voraussetzungen, dem sozialen Status, Schulabschlüssen und dem Geschlecht denkbar (vgl. Becker, 2011; Granato, 2013; Schmidt-Koddenberg \& Zorn, 2012). Einen Erklärungsansatz liefert die in Migrantenfamilien vergleichsweise geringere konkrete Unterstützung bei Schul- und Ausbildungsfragen ${ }^{4}$, die Jugendliche beispielsweise durch Hilfe bei den Hausaufgaben erhalten (Boos-Nünning \& Karakaşoğl, 2004) ${ }^{5}$. Dieses Phänomen kann teilweise durch eine mögliche mangelnde Erfahrung von Migranteneltern

\footnotetext{
${ }^{4}$ Leenen, Grosch und Kreidt (1990) fassen darin die „Grundlegung von Basiskompetenzen und -motivationen bis zur Bildungslaufbahnberatung und Biographieplanung, von der Sicherung adäquater Lern- und Arbeitsbedingungen bis zur konkreten schulischen Lernunterstützung“ zusammen (S. 753).

${ }^{5}$ Beicht und Granato (2011) betonen jedoch, dass junge Migrant*innen eine starke emotionale Unterstützung durch ihre Familie bei Bildungsfragen erhalten.
} 
mit dem deutschen Bildungs- und Übergangssystem erklärt werden (Becker, 2011; Kuhnke \& Müller, 2009). Ähnlich folgerte Stürzer (2014), dass Unterschiede in den Schulerfolgen Lernender verschiedener Herkunftsländer auch in den (durch die Eltern) mitgebrachten Bildungsvoraussetzungen zu suchen sind. Weitere Ursachen der geringeren Erfolge bei der Ausbildungssuche von Migrant*innen können zudem in den Auswahlprozessen der Unternehmen selbst, also durch „,Diskriminierungsprozesse seitens der Arbeitgeber" (Diehl et al., 2009, S. 48) vermutet werden (vgl. zsf. Granato, 2013). Cremers (2012) richtet das Augenmerk zusätzlich auf die Möglichkeit, dass das „Qualifikationspotenzial von Schülerinnen und Schülern ohne deutsche Staatsangehörigkeit am wenigsten angemessen entwickelt" (S. 33) wird.

Obwohl Beckers (2011) empirische Untersuchungen zumindest , die statistische Diskriminierung von Migrantenkindern in der Schule“ (S. 21) widerlegen, räumt er gleichzeitig ein, dass sich schlechtere Deutschnoten zu Ungunsten von Lernenden mit Migrationshintergrund auf die Schulübergangsentscheidungen auswirken. Bei gleichem Leistungsniveau entscheiden sich ihre Eltern im Vergleich zu einheimischen Familien häufiger für den Besuch der Hauptschule (Becker, 2011). Auch Bennett (2007) wies empirisch nach, dass für amerikanische High-SchoolSchüler*innen Englisch als Zweitsprache ein Nachteil für das Erreichen eines hohen Grads an Berufsorientierung darstellt. Daran anknüpfend wird hier folgend der Zusammenhang zwischen der deutschen Muttersprache, als Indikator der Kompetenz in der Landessprache, den besuchten BO-Maßnahmen und der persönlich empfundenen Berufswahlvorbereitung Heranwachsender analysiert.

\section{Empirische Studien zu Effekten von Berufsorientierungsmaßnahmen}

Eine grundsätzliche Herausforderung bei der Effektmessung von BO-Maßnahmen ist die Heterogenität der Formate, die unter dem gleichen Begriff zusammengefasst und durchgeführt werden können. Die Spannweite des Formats Potentialanalyse beispielsweise erstreckt sich in Abhängigkeit der Anbieter und lokalen Vorgaben vom schriftlichen Wissens- und Interessenstest bis hin zum mehrtägigen Workshop mit Rollenspielen und praktischen Arbeitsproben. Bisherige Studien zu BO-Maßnahmen, wie die Analyse von Ratschinski und Struck (2016) zu Effekten der Interventionen Potentialanalyse, Praktikum und Werkstatttage auf die Berufswahlkompetenz, konnten diese nur in geringem Maße nachweisen. Ähnlich zeigte sich in einer Studie an amerikanischen High-Schools ein positiver, aber nur geringer Effekt des unbezahlten Pflichtpraktikums auf die Berufsorientierung. Für ein zweites, bezahltes Pflichtpraktikum waren in der gleichen Studie jedoch keine signifikanten Effekte ersichtlich (Bennett, 2007). Für Hauptschulabgänger*innen kann die Teilnahme an „Berufsstarter- und Praxisklassen“ mit „,wöchentlichen 
betrieblichen Praxistagen“ (Kohlrausch \& Solga, 2012, S. 754) zu einer höheren Erfolgsquote beim Übergang in eine ,,voll qualifizierte Ausbildung“ (Kohlrausch \& Solga, 2012, S. 766) als beim Besuch von Regelklassen führen.

\subsection{Forschungsfragen}

In dieser Studie wird der Frage nachgegangen, ob Zusammenhänge zwischen persönlichen Merkmalen Heranwachsender und den von ihnen besuchten schulischen BO-Maßnahmen in Bezug auf ihre persönlich empfundene Berufswahlvorbereitung bestehen. Aus dieser Überlegung lassen sich die folgenden Forschungsfragen ableiten:

(1) Stehen die persönlichen Merkmale (Geschlecht, Muttersprache und Schulleistung) mit dem subjektiven Stand der Berufswahlvorbereitung in Zusammenhang?

(2) Welchen Effekt haben BO-Maßnahmen auf die durch die Jugendlichen empfundene Berufswahlvorbereitung? Verschiedene Studien haben bereits die Einzeleffekte des Geschlechts, der Muttersprache und der Schulleistung untersucht, sodass hier des Weiteren die Frage nach Interaktionseffekten beantwortet werden soll:

(3) Beurteilen Jugendliche aufgrund unterschiedlicher persönlicher Merkmale ihre subjektive Berufswahlvorbereitung unterschiedlich stark in Abhängigkeit der Teilnahme an den betrachteten BO-Maßnahmen?

\section{$9.4 \quad$ Methode}

\section{Beschreibung der Stichprobe}

Für die vorliegende Studie wurde der Datensatz des zweiten Messzeitpunktes der Berliner Berufs- und Studienorientierungsstudie (BeBest-Studie) verwendet (vgl. Ohlemann et al., 2016, siehe auch Abschnitt 8.1). An dem Paper-Pencil-Fragebogen nahmen $N=1698$ Schüler*innen im Alter von 10 bis 18 Jahren der achten bis zehnten Jahrgangsstufe teil. Die Datenerhebung erfolgte im Herbst 2014 an 14 für Berlins Schullandschaft repräsentativen Integrierten Sekundarschulen ${ }^{6}$. Von den

\footnotetext{
${ }^{6}$ Die Integrierte Sekundarschule (ISS) ist seit dem Schuljahr 2010/11 neben dem Gymnasium die zweite reguläre Schulform im allgemeinbildenden Sekundarbereich in Berlin. Die leistungsbezogene Binnendifferenzierung und die starke Praxisorientierung bilden die zentralen
} 
Lernenden, die Angaben zu ihrem Geschlecht und ihrer Muttersprache machten, waren $n=737$ Mädchen (44\%). 958 Teilnehmende (57\%) gaben Deutsch als ihre Muttersprache an. Im Mittel erreichten die Lernenden im letzten Jahreszeugnis vor der Befragung in den Fächern Deutsch, Englisch und Mathematik einen selbstberichteten Notendurchschnitt von 3,1 auf der Notenskala. Die Teilnahme an den BO-Maßnahmen konnte einzeln beantwortet werden, sodass sich unterschiedliche Grundgesamtheiten ergeben (siehe Tabelle 9.1).

\section{Instrumente}

Für die vorliegenden Analysen wurde die von Seifert und Bergmann (1992) stammende, adaptierte Skala Subjektiver Stand in der Berufswahlvorbereitung (B. Kracke, 2001, zit. n. Hany \& Driesel-Lange, 2006) verwendet. Anhand einer vierstufigen Skala $(0=$ stimmt überhaupt nicht, $3=$ stimmt genau $)$ konnten die Lernenden sechs Aussagen beurteilen. Beispielsweise ,Ich weiß nicht, wo ich genauere Informationen über Studien- und Berufsmöglichkeiten bekommen kann, die für mich in Frage kommen“. Negativ formulierte Items wurden entsprechend umkodiert. Döring und Bortz (2016) sehen die interne Skalenkonsistenz mit einem Wert für Cronbachs Alpha von $\alpha>.8$ als gesichert an. Zu Gunsten einer höheren internen Konsistenz wurde das Item ,Ich fühle mich bisher noch nicht ausreichend auf meine Studienund Berufswahl vorbereitet" aus der Skala entnommen, die dann einen Wert von $\alpha=.75$ erreicht. Aktivitäten zur Berufswahl in der Schule wurden anhand der Skala Schulische Maßnahmen zur Berufsorientierung von B. Kracke und SchmittRodermund (2001) erfasst. Die Skala wurde um acht BO-Maßnahmen auf insgesamt 25 Items erweitert. Die Lernenden beantworteten die Frage der Teilnahme $(0=$ keine Teilnahme, 1 = Teilnahme) für jede BO-Maßnahme einzeln.

Außerdem wurden die Teilnehmenden gebeten, ihr Geschlecht ( $0=$ männlich, $1=$ weiblich), ihre Muttersprache ( $1=$ Deutsch, $0=$ andere Sprache), sowie ihre Zeugnisnoten ( 1 = sehr gut, $6=$ ungenügend) für die Fächer Deutsch, Englisch und Mathematik des letzten Zeugnisses (Schuljahr 2013/14) als Indikator der Schulleistung anzugeben. Aus den Angaben für die drei Fächer wurde eine Durchschnittsnote errechnet und diese anschließend rekodiert, sodass 1 die schwächste Leistung und 6 die beste Leistung repräsentieren.

\section{Analysemodell, Modellprüfung und Effektstärken}

Um die Relevanz der oben dargestellten BO-Maßnahmen zu analysieren, wurden für die informierenden und praxisorientierten Angebote zwei separate, lineare

inhaltlichen und methodischen Abgrenzungen zum Gymnasium (vgl. Bartels \& Nix, 2010; Senatsverwaltung für Bildung, Wissenschaft und Forschung Berlin, 2010). 
Regressionen durchgeführt. Dabei wurden im ersten Schritt die unabhängigen Variablen Geschlecht, Muttersprache und Schulleistung und im zweiten Schritt jeweils alle informierenden beziehungsweise praxisorientierten Maßnahmen in das Modell

Tabelle 9.1 Teilnahme an den schulischen BO-Maßnahmen

\begin{tabular}{|c|c|c|}
\hline \multirow[t]{2}{*}{ BO-Maßnahme } & \multicolumn{2}{|l|}{ Teilnahme } \\
\hline & in absoluten Zahlen & in $\%$ \\
\hline \multicolumn{3}{|l|}{ Informierende Maßnahmen } \\
\hline Berufskundliche Bücher \& Broschüren & 481 & $31 \%$ \\
\hline Berufskundliche Filme, TV- /Radiosendung & 327 & $21 \%$ \\
\hline Besuch im BIZ* & 609 & $39 \%$ \\
\hline Betriebsbesichtigungen & 600 & $39 \%$ \\
\hline Gespräche mit Berufsberater* & 550 & $36 \%$ \\
\hline Internetrecherche & 677 & $43 \%$ \\
\hline Jobmesse* & 572 & $36 \%$ \\
\hline Kontakt zu Berufsverbänden/Kammern & 124 & $8 \%$ \\
\hline Studienberatung & 211 & $13 \%$ \\
\hline Tag der offenen Tür (Unternehmen) & 437 & $28 \%$ \\
\hline Tag der offenen Tür (Hochschule) & 236 & $15 \%$ \\
\hline Vorträge von der Berufsberatung & 596 & $39 \%$ \\
\hline \multicolumn{3}{|l|}{ Praxisorientierte Maßnahmen } \\
\hline Assessmentcenter-Übung & 195 & $13 \%$ \\
\hline Bewerbungstraining* & 778 & $50 \%$ \\
\hline Girls-Day/Boys-Day & 617 & $39 \%$ \\
\hline Ferien- oder Nebenjob & 352 & $22 \%$ \\
\hline Potentialanalyse*a & 706 & $46 \%$ \\
\hline Praktikum* & 889 & $56 \%$ \\
\hline Praxislernen/Praxislerntage* & 368 & $23 \%$ \\
\hline Projektlerntage innerhalb/außerhalb der Schule & 594 & $38 \%$ \\
\hline Schnupperstudium & 140 & $9 \%$ \\
\hline Andere Angebote von Hochschulen (z. B. Schülerlabor) & 124 & $8 \%$ \\
\hline Schülerfirma & 157 & $10 \%$ \\
\hline
\end{tabular}


Tabelle 9.1 (Fortsetzung)

\begin{tabular}{l|l|l}
\hline \multirow{2}{*}{ BO-Maßnahme } & \multicolumn{2}{l}{ Teilnahme } \\
\cline { 2 - 3 } & in absoluten Zahlen & in \% \\
\hline Werkstattarbeit innerhalb der Schule & 823 & $52 \%$ \\
\hline Werkstattarbeit außerhalb der Schule & 379 & $24 \%$ \\
\hline
\end{tabular}

Anmerkung. *Angebote, die in der ersten Regression signifikante Effekte auf den subjektiven Stand der Berufswahlvorbereitung zeigten (siehe nächster Abschnitt.)

${ }^{a}$ Unter dem Begriff Potentialanalyse wurden in Berlin zum Zeitpunkt der Erhebung verschiedene Aufgabenformate zur Identifikation persönlicher Interessen und Fähigkeiten zusammengefasst, die mit Abstrichen untereinander vergleichbar sind.

integriert. Als abhängige Variable wurde der subjektive Stand der Berufswahlvorbereitung herangezogen. Für die sieben BO-Maßnahmen mit einem signifikanten Effekt wurde im Anschluss je eine vierstufige, lineare Regression durchgeführt. In diesen Regressionen wurden, wie in Abbildung 9.1 beispielhaft dargestellt ist, zunächst die unabhängigen Variablen Geschlecht, Muttersprache und Schulleistung, dann die jeweilige BO-Maßnahme und schließlich die Produktterme der Zweifach- beziehungsweise Dreifachinteraktionen in das Modell eingegeben. Nach Field (2009) wurden die Daten auf mögliche Ausreißer und das Modell bezogen auf die getroffenen Annahmen ${ }^{7}$ hin geprüft. Zur Darstellung der Haupt- und Interaktionseffekte werden die standardisierten Betakoeffizienten genutzt. Dabei stehen Werte zwischen null und eins für einen positiven Effekt auf die abhängige Variable, Werte unter null bedeuten einen negativen Effekt. Als Effektmaß des Gesamtmodells wird der korrigierte Determinationskoeffizient $R^{2}$ betrachtet, dessen Effektstärke nach Cohen (1988) für multivariate Analysen bei $R^{2}$-Werten von 0.0196 als klein, ab 0.13 als mittelmäßig und ab Werten von 0.26 als groß eingestuft wird.

\footnotetext{
${ }^{7}$ In Anlehnung an eine Simulationsstudie von Lumley et al. (2002) wurde die multiple lineare Regression trotz der festgestellten Heteroskedastizität der Daten durchgeführt.
} 


\begin{tabular}{|c|c|c|c|}
\hline Schritt 1 & Schritt 2 & Schritt 3 & Schritt 4 \\
\hline $\begin{array}{l}\text { Sozioökonomische } \\
\text { Faktoren } \\
\text { - Geschlecht } \\
\text { - Muttersprache } \\
\text { - Schulleistung }\end{array}$ & $\begin{array}{l}\text { BO-Maßnahme } \\
\text { - Praktikum }\end{array}$ & $\begin{array}{l}\text { Zweifachinteraktionen } \\
\text { - PraktikumGeschlecht } \\
\text { - PraktikumMuttersprache } \\
\text { - PraktikumSchulleistung } \\
\text { - GeschlechtMuttersprache } \\
\text { - GeschlechtSchulleistung } \\
\text { - Muttersprache*Schulleistung }\end{array}$ & $\begin{array}{l}\text { Dreifachinteraktionen } \\
\text { - PraktikumGeschlecht*Muttersprache } \\
\text { - PraktikumGeschlecht }{ }^{\star} \text { Schulleistung } \\
\text { - PraktikumMuttersprache }{ }^{\star} \text { Schulleistung }\end{array}$ \\
\hline \multicolumn{4}{|c|}{ Subjektiver Stand der Berufsvorbereitung } \\
\hline
\end{tabular}

Abbildung 9.1 Modellübersicht zur vierstufigen, linearen Regression am Beispiel Praktikum

\subsection{Ergebnisse}

\section{Ergebnisse zu Geschlechterunterschieden}

Das Geschlecht der Jugendlichen zeigte in allen sieben Regressionsmodellen signifikante Effekte auf die persönlich empfundene Berufswahlvorbereitung der Heranwachsenden (siehe Tabelle 9.2, 9.3, 9.4, 9.5, 9.6, 9.7 und 9.8, jeweils Zeile 4): Jungen schätzten ihre persönliche Berufswahlvorbereitung tendenziell höher ein, als Mädchen dies taten. Für die Mehrzahl der betrachteten Modelle liegt die Effektstärke des Geschlechts zwischen $\beta=-.08$ und -.09 .

Im Modell zum Bewerbungstraining (vgl. Tabelle 9.6) wurden die größten Effektstärken $\left(\beta=-.16^{*}\right)$ und damit die größten Unterschiede zwischen der Selbsteinschätzung von Jungen und Mädchen gemessen. Nach Einführung der Interaktionsterme in die Modelle lassen sich zwei Tendenzen beobachten. In der Hälfte der Fälle verliert das Geschlecht als einzeln betrachteter Faktor seine Signifikanz, der Effekt auf die persönlich empfundene Berufswahlvorbereitung ist statistisch gesehen also nicht mehr bedeutsam. Für die andere Hälfte der Fälle, nämlich für Gespräche mit dem Berufsberater, das Bewerbungstraining und das Praxislernen bleibt der Einfluss der Geschlechterunterschiede auch neben den Interaktionstermen bestehen (vgl. Tabelle 9.3, $9.6 \& 9.8$ jeweils Zeile 4). 


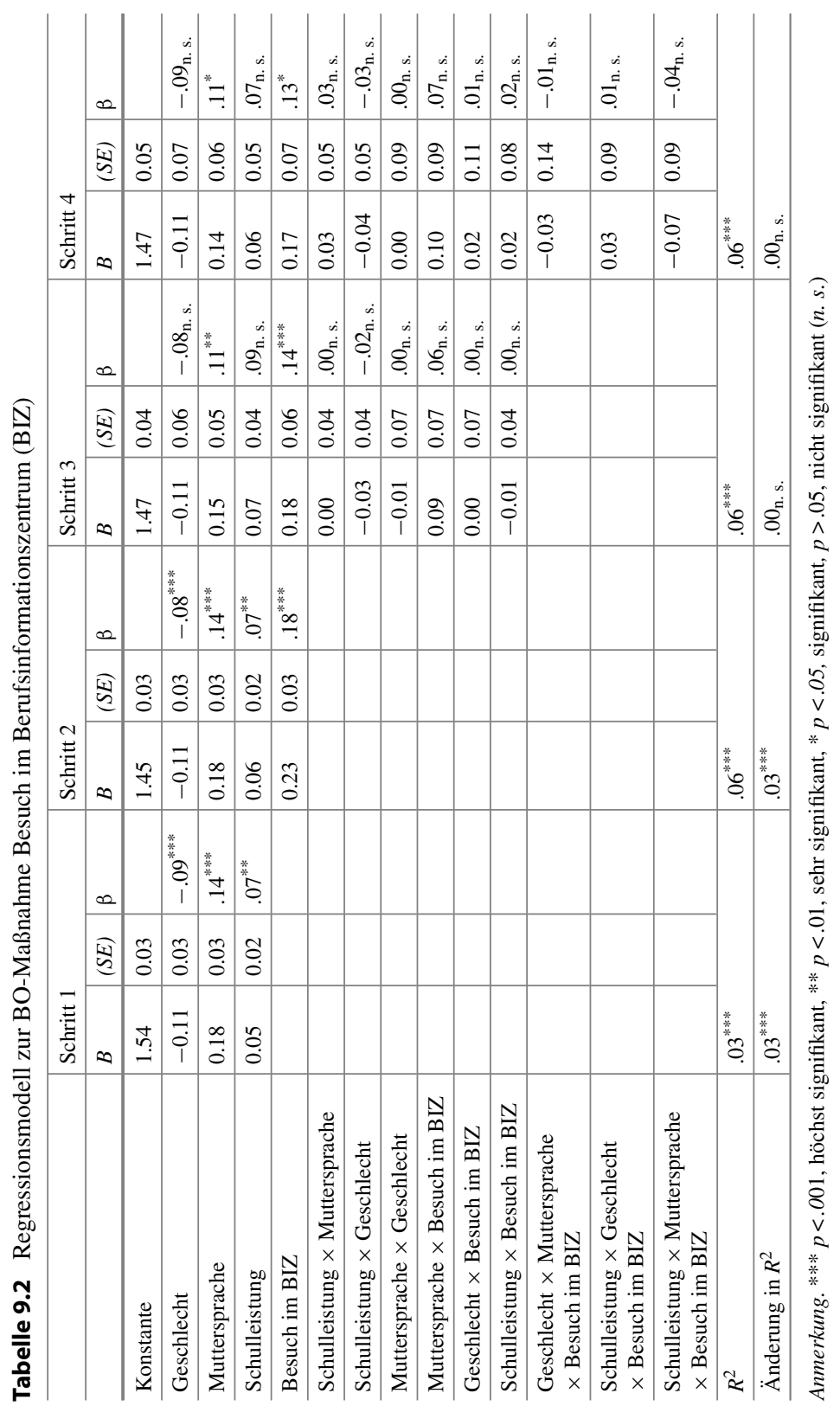




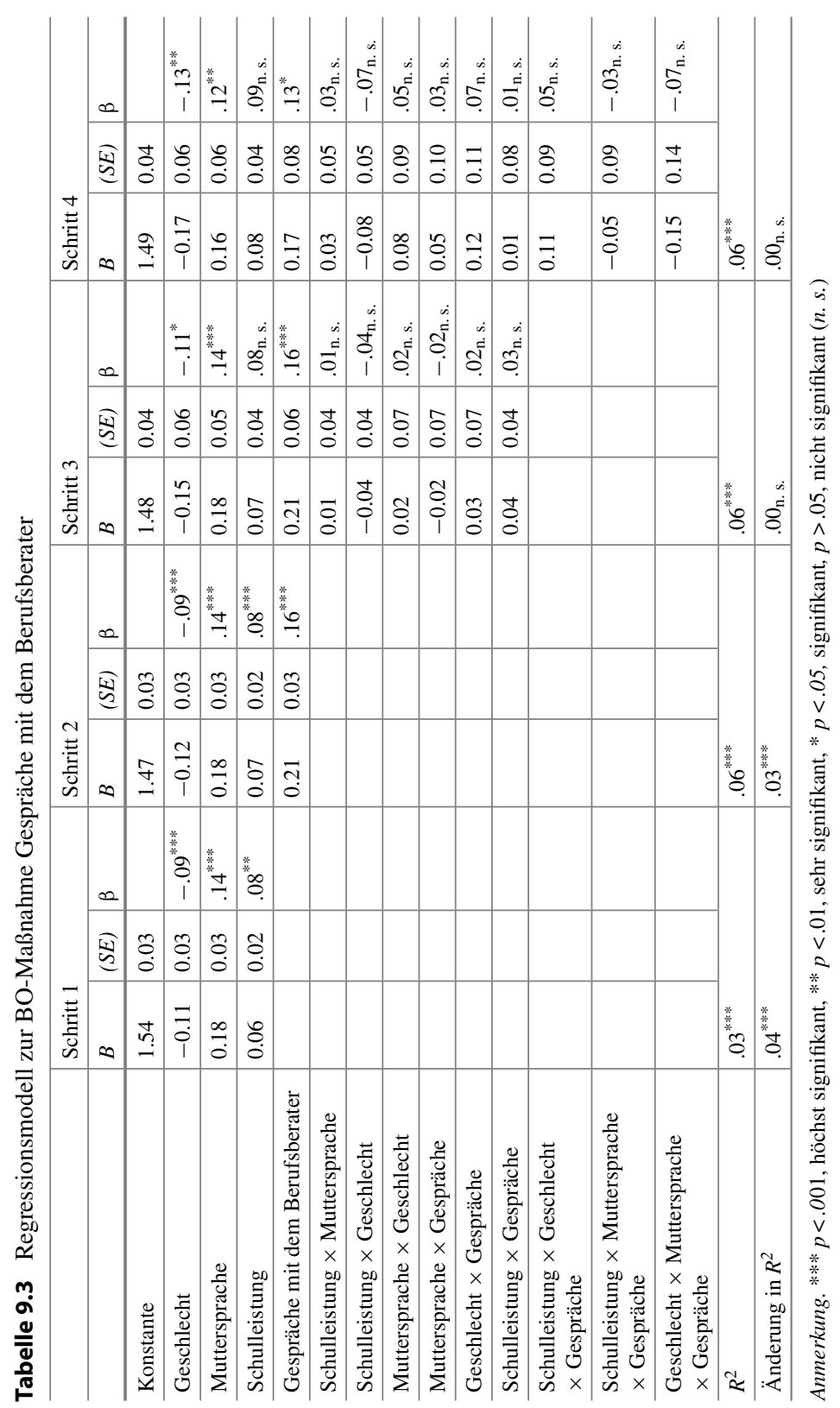




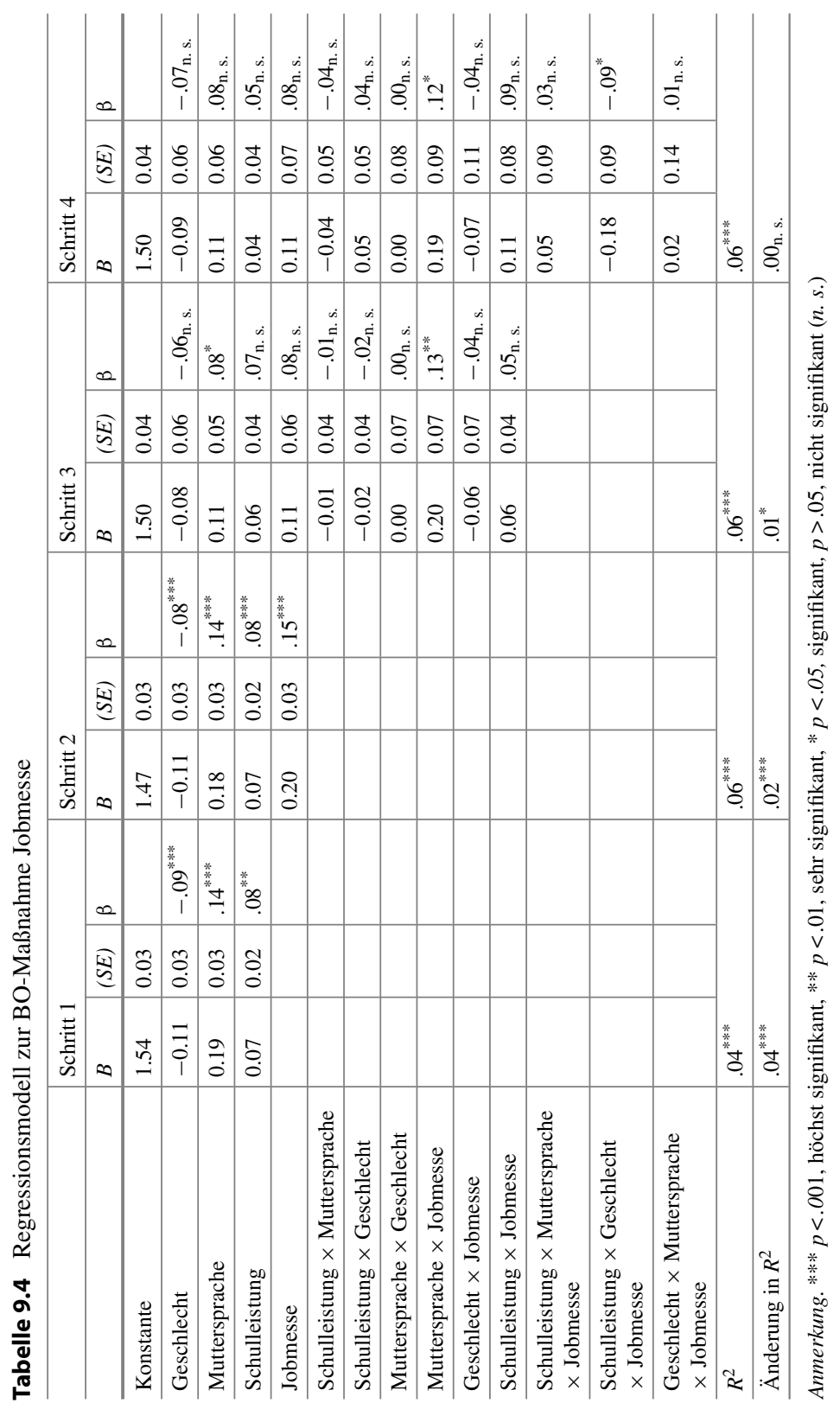




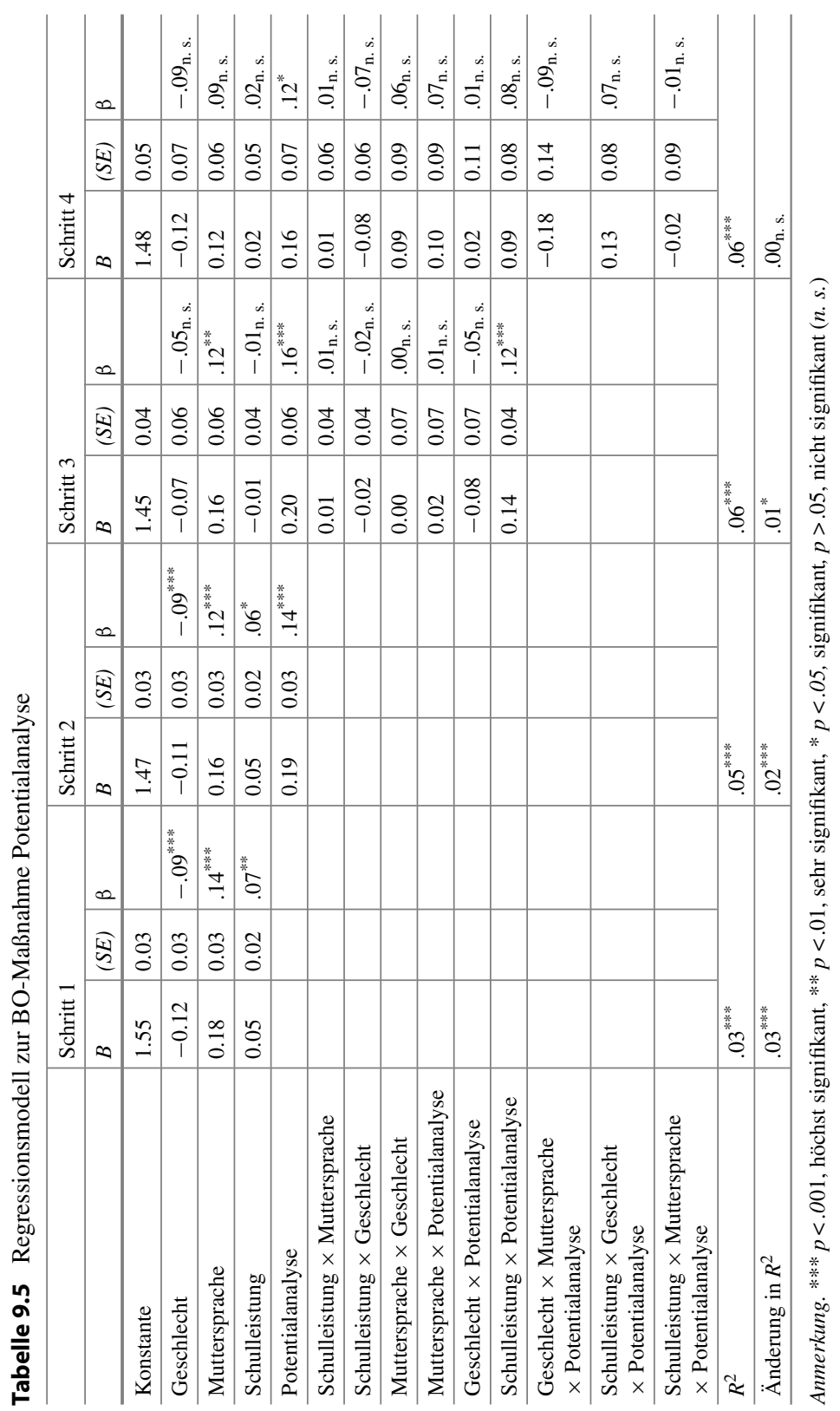




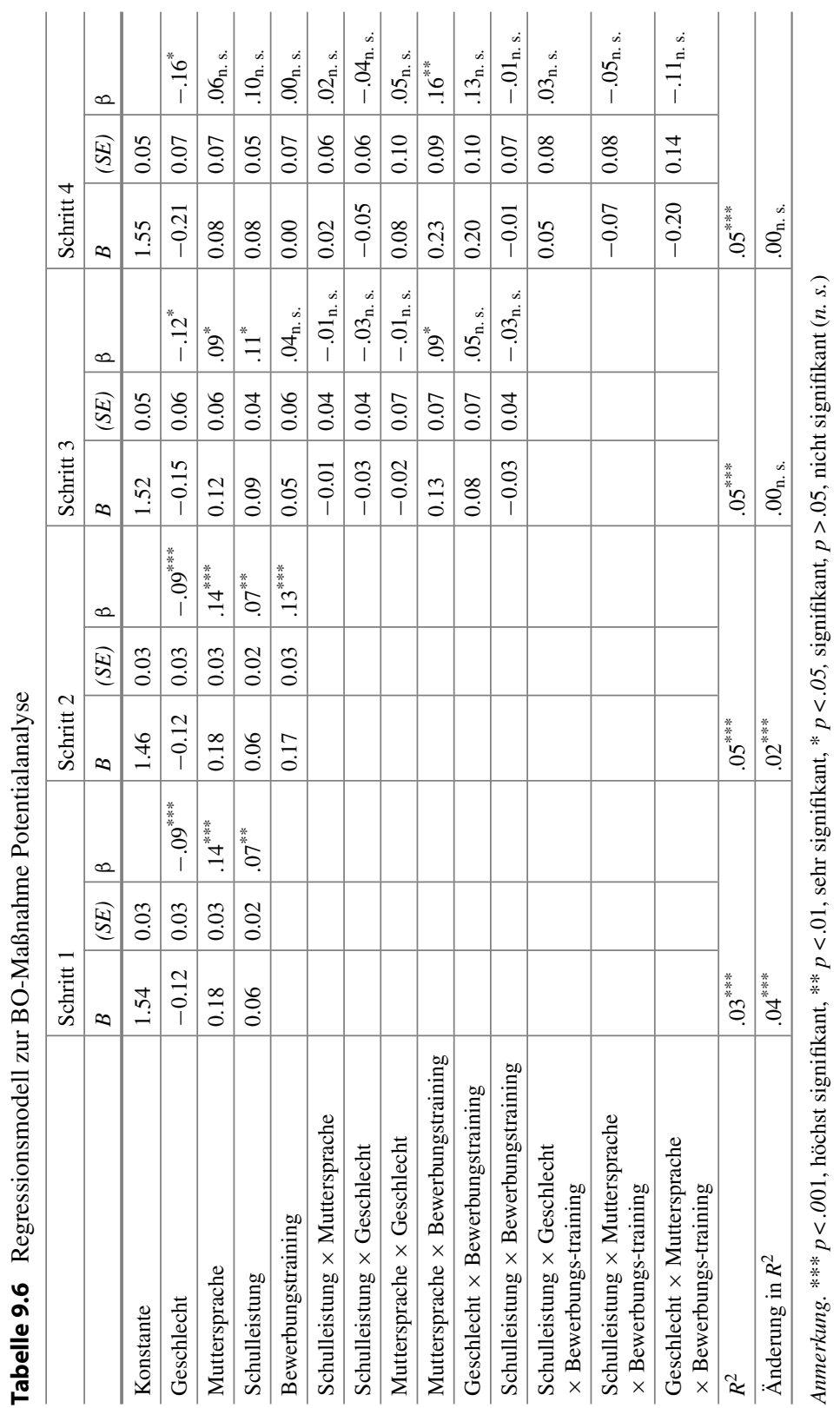




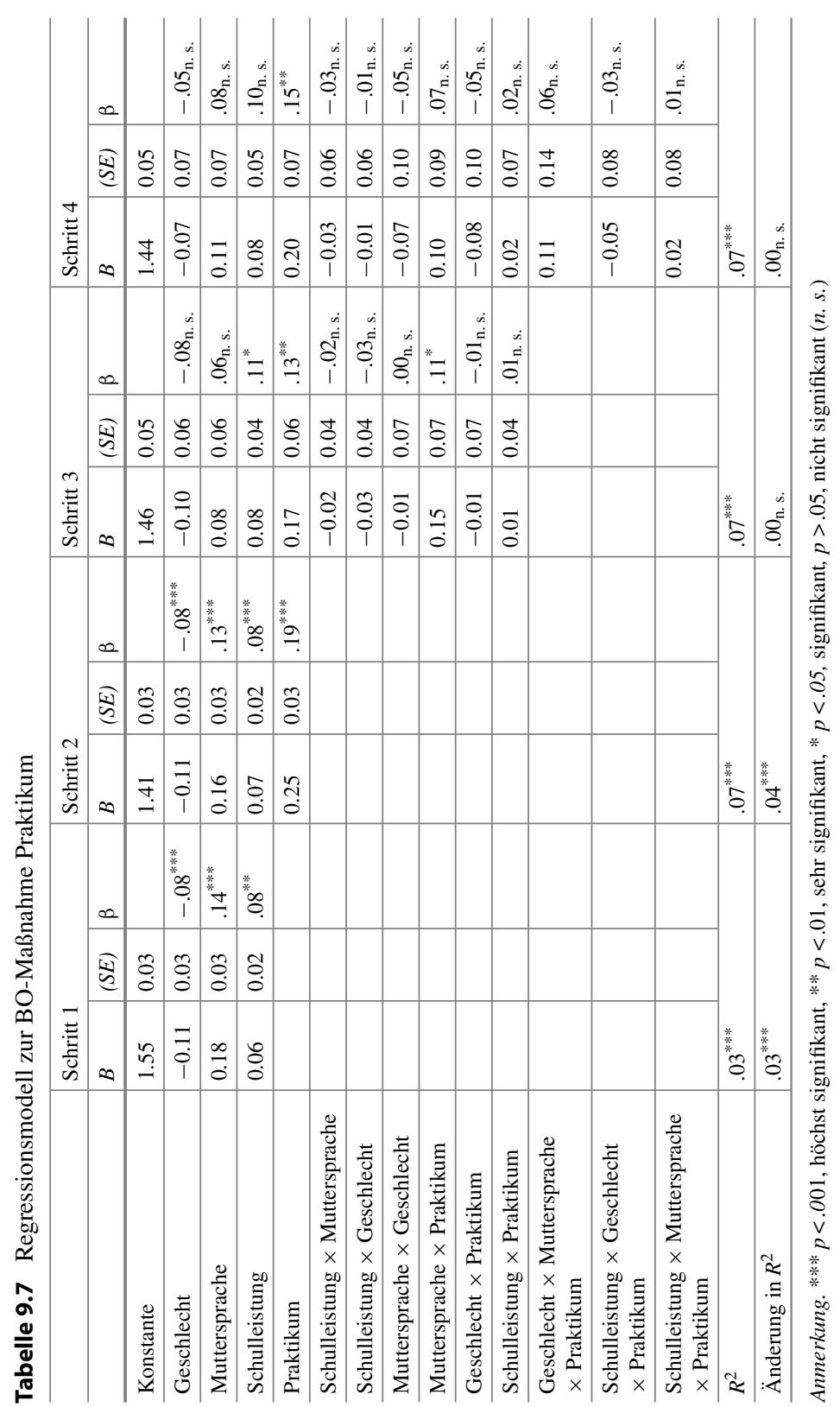




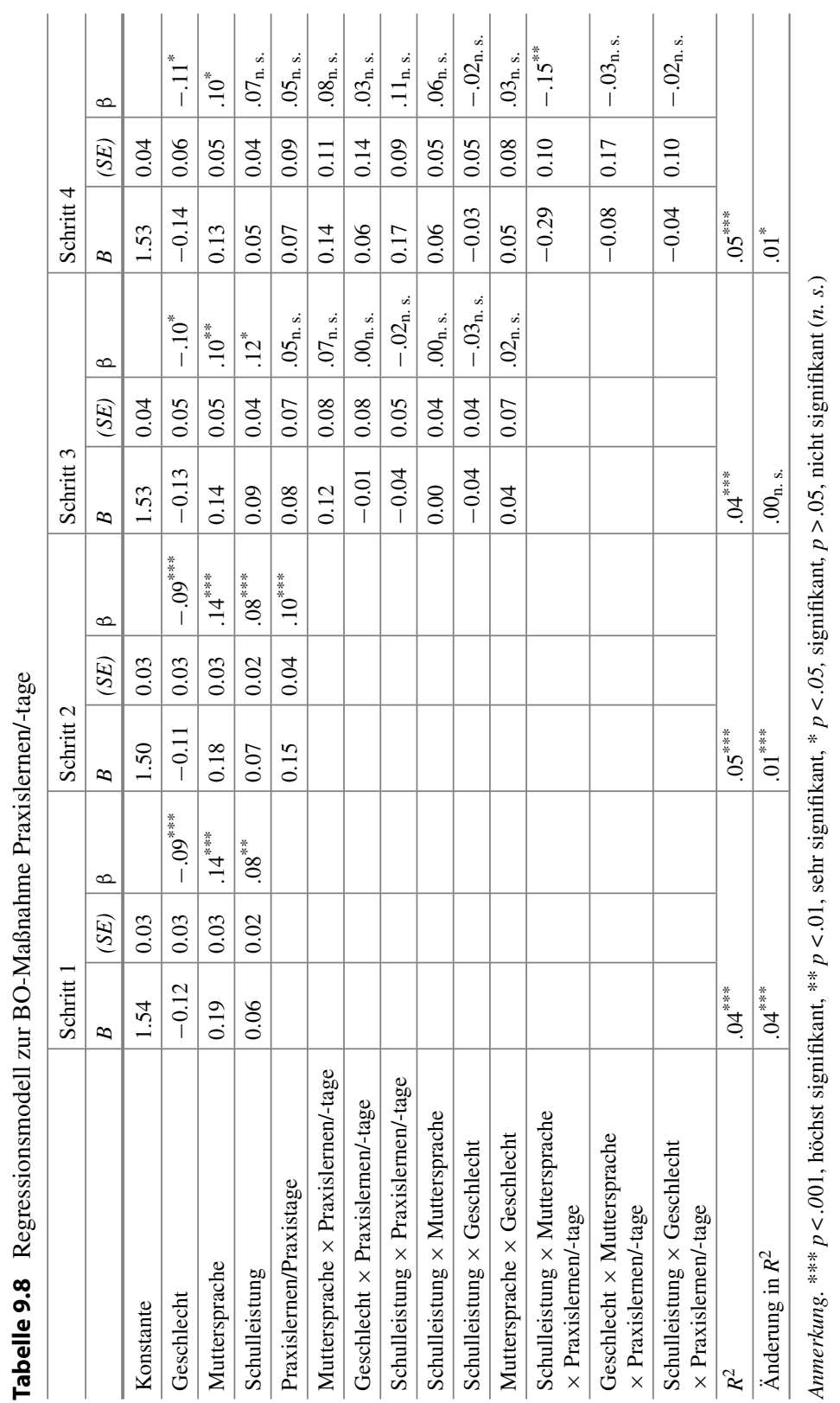




\section{Ergebnisse zur Muttersprache}

Auch die Muttersprache führt in allen Modellen zu signifikanten Unterschieden in der Wahrnehmung der Berufswahlvorbereitung. Heranwachsende mit deutscher Muttersprache erleben sich selbst im Durchschnitt als besser vorbereitet als ihre anderssprachigen Peers. Unabhängig des betrachteten Modells ist der Effekt der Muttersprache im Vergleich zum Geschlecht stabiler und größer. Sie trägt also noch stärker zu den empfundenen Unterschieden bei. In allen Modellen erreicht die Muttersprache $\beta$-Werte bis zu .14 und verliert nur im Zusammenhang mit dem Praktikum nach Zugabe der Zweifachinteraktionsterme an Signifikanz (vgl. Tabelle 9.7). Allerdings nimmt der Effekt der Muttersprache, ähnlich wie der des Geschlechts, mit zunehmender Modellkomplexität ab.

\section{Ergebnisse zur Schulleistung}

Die Schulleistung der Lernenden, gemessen an den durchschnittlichen Zeugnisnoten der Unterrichtsfächer Deutsch, Mathematik und Englisch des letzten Halbjahres, zeigte in allen Berechnungen einen signifikanten Effekt. Schüler*innen schätzten ihre Berufswahlvorbereitung mit steigenden schulischen Leistungen höher ein. Diese Aussage muss jedoch insofern eingegrenzt werden, als dass die Effektstärke ( $\beta$ $=.06-.09$ ) und damit der Einfluss auf die persönlich empfundene Berufswahlvorbereitung auch im Vergleich zu denen der Haupteffekte Geschlecht und Muttersprache als gering einzustufen ist. Wie bereits bei den Faktoren Geschlecht und Muttersprache teilweise zu beobachten war, verliert der Effekt der Schulleistung spätestens nach Einführung der Dreifachinteraktionen seine Signifikanz. Jedoch findet sich die Schulleistung als Komponente mehrerer signifikanter Interaktionen wieder (vgl. übernächster Abschnitt).

\section{Ergebnisse zu den BO-Maßnahmen}

Alle sieben hier untersuchten BO-Maßnahmen haben einen signifikanten positiven Effekt auf den subjektiven Stand der Berufswahlvorbereitung der teilnehmenden Lernenden gezeigt. Jugendliche, die die jeweilige Maßnahme besucht hatten, schätzten ihre Vorbereitung im Durchschnitt als fortgeschrittener ein als Jugendliche, die nicht an der jeweiligen Intervention teilgenommen hatten. Im Folgenden werden die Ergebnisse detaillierter dargestellt. Es zeigten sich wieder zwei generelle Tendenzen: Die Hälfte der BO-Maßnahmen hatte einen positiven Effekt auf die persönlich empfundene Berufswahlvorbereitung, der jedoch nach Hinzufügen der Interaktionsterme seine Signifikanz verliert. Zu diesen Interventionen gehören der Besuch einer Jobmesse $\left(\beta=.15^{* * *}\right)$, die Teilnahme an einem Bewerbungstraining $\left(\beta=.13^{* * *}\right)$, sowie die Teilnahme an Praxistagen $\left(\beta=.10^{* * *}\right)$. Alternativ blieben die Effekte der BO-Maßnahmen unter leichter Degression der Effektstärke auch im Zusammenspiel 
mit den Zweifach- und Dreifachinteraktionen signifikant. Der Einfluss dieser vier Maßnahmen war in mindestens einem Modellschritt größer als der der Interventionen aus der zuvor beschriebenen Maßnahmengruppe. Die Maßnahmen Besuch im Berufsinformationszentrum (BIZ) $\left(\beta=.18^{* * *}, .14^{* * *}\right.$ bzw. .13*), Gespräche mit dem Berufsberater $\left(\beta=.16^{* * *}, .16^{* * *}\right.$ bzw. $\left..13^{*}\right)$, Potentialanalyse $\left(\beta=.14^{* * *}\right.$, $.16^{* * *}$ bzw. $\left..12^{*}\right)$ und Praktikum $\left(\beta=.19^{* * *}, .13^{* *}\right.$ bzw. $\left..15^{* *}\right)$ scheinen folglich einen stärkeren und stabileren Einfluss auf die empfundene Berufswahlvorbereitung der Jugendlichen zu nehmen. Alle sieben untersuchten BO-Maßnahmen zeigten im Ganzen stärkere Effekte als die betrachteten sozioökonomischen Faktoren Geschlecht und Muttersprache beziehungsweise die Schulleistung.

\section{Ergebnisse zu den Interaktionseffekten}

Mehrere Interaktionen zwischen einzelnen BO-Maßnahmen und den persönlichen Merkmalen hatten signifikante Effekte auf die subjektiv wahrgenommene Berufswahlvorbereitung der teilnehmenden Jugendlichen. Dabei scheint insbesondere das Zusammenspiel mit der Muttersprache von Relevanz zu sein. Auch in direkter Interaktion mit einer BO-Maßnahme übt die deutsche Muttersprache einen günstigen Einfluss auf die gefühlte Berufswahlvorbereitung der Heranwachsenden aus. Deutschsprachige Lernende, die eine Jobmesse besucht hatten, schätzten ihre persönliche Berufswahlvorbereitung höher ein als ihre Vergleichsgruppe ohne den Besuch der Jobmesse $\left(\beta=.13^{* *}\right.$ bzw. .12*, siehe Tabelle 9.4, Zeile 11).

Auch bei deutschsprachigen Teilnehmer*innen eines Bewerbungstrainings beobachten wir diesen Effekt $\left(\beta=.09^{*}\right.$ bzw. .16** , siehe Tabelle 9.6, Zeile 9). Dieser muss jedoch unter Einschränkungen interpretiert werden, da die Veränderungen in der Varianzaufklärung des Gesamtmodells nach Hinzufügen der Interaktionen nicht (mehr) signifikant sind. Der Effekt zwischen der Muttersprache und dem Praktikum ( $\beta=.11^{*}$, siehe, Zeile 9) muss mit einem ähnlichen Vorbehalt interpretiert werden. Die Wirkrichtung scheint wieder die gleiche zu sein, sodass deutschsprachige Jugendliche nach der Durchführung eines Praktikums einen besonders starken Effekt auf ihre Berufswahlvorbereitung spürten. Für anderssprachige Jugendliche konnten für die genannten BO-Maßnahmen keine signifikanten Effekte beobachten werden.

Im Zusammenspiel mit dem Praxislernen besteht indes eine signifikante Dreifachinteraktion ( $\beta=-.15^{* *}$, siehe Tabelle 9.8, Zeile 14) mit der Muttersprache und der Schulleistung der Lernenden: Demzufolge profitieren deutschsprachige Schüler*innen mit vergleichsweise schwachen bis durchschnittlichen Schulleistungen vom Praxislernen. Auch anderssprachige Jugendliche gewinnen durch die Teilnahme am Praxislernen. Allerdings trifft diese Aussage bei ihnen auf die Gruppe der überdurchschnittlich guten Schüler*innen zu. Beide Gruppen schätzten ihre 
subjektive Berufswahlvorbereitung mit dem Maßnahmenbesuch höher ein als ihre Peers im Regelunterricht. Die Schulleistung hat, neben der gerade beschriebenen Dreifachinteraktion, auch in Interaktion mit der Potentialanalyse einen signifikanten Effekt ( $\beta=.12^{* * *}$, siehe Tabelle 9.5, Zeile 13): Schüler*innen mit vergleichsweise hohen Leistungen profitieren demnach besonders von der Potentialanalyse.

Betrachtet man die Interaktion zwischen der Schulleistung, dem Geschlecht und dem Besuch einer Jobmesse ( $\beta=-.09^{*}$, siehe Tabelle 9.4, Zeile 15) lässt sich beobachten, dass Jungen mit vergleichsweise überdurchschnittlichen Schulleistungen in besonderem Maße von dem Besuch einer Jobmesse profitieren. Sie bewerten ihre Berufswahlvorbereitung höher als Jungen mit einem ähnlichen Notenprofil, die keine Karrieremesse besucht hatten. Die Veränderung in der Signifikanzaufklärung des Gesamtmodells ist jedoch nach Hinzufügen der Dreifachinteraktionen nicht mehr signifikant, sodass der Effekt nur mit Einschränkungen, also höchstens als tendenzieller Indikator, interpretiert werden kann. Der Faktor Geschlecht wurde bezogen auf Interaktionseffekte nur in dieser Kombination sichtbar.

\subsection{Diskussion}

Das Ziel der hier dargestellten Analysen war die systematische Untersuchung möglicher Effekte von Geschlecht, Muttersprache und Schulleistung sowie der BO-Maßnahmen auf die subjektiv empfundene Berufswahlvorbereitung von Schüler*innen der Sekundarstufe I. Ferner sollten mögliche Interaktionseffekte betrachtet werden. Deutlich wurde, dass die subjektive Berufswahlvorbereitung bei den verschiedenen sozioökonomischen Gruppen unterschiedlich stark ausgeprägt ist und sieben BO-Maßnahmen, nämlich der Besuch im BIZ, das Gespräch mit einem Berufsberater, die Potentialanalyse, das Bewerbungstraining, das Praktikum, das Praxislernen und der Besuch einer Jobmesse, einen signifikanten positiven Effekt auf die subjektive Berufswahlvorbereitung der Teilnehmenden hatten. Allerdings weisen die untersuchten Variablen und ihre Interaktionsterme geringe Effektstärken auf und erklären nur einen kleinen Teil der Varianz am Gesamtmodell, also am Zustandekommen der subjektiven Berufswahlvorbereitung. So sind die betrachteten Faktoren alleinstehend nicht ausreichend, um als Basis zur Differenzierung schulischer Berufsorientierung zu dienen. Bezogen auf den aktuellen Forschungsstand zeigen die erzielten Ergebnisse Parallelen $\mathrm{zu}$ früheren Studien, in denen ebenfalls nur geringe Effektstärken der BOMaßnahmen gemessen werden konnten. Dabei stellt sich weiterhin die Frage, ob 
die Effekte generell nur in kleinem Maße bestehen oder ob alternative Messverfahren diese besser sichtbar machen könnten. Des Weiteren konnte der Einfluss des Geschlechts und der schulischen Leistung in der Berufswahl bestätigt und weitere Effekte der Muttersprache gezeigt werden (vgl. Busch, 2013; Cremers, 2012; Fend, 1991).

\section{Geschlechterunterschiede in der wahrgenommenen Berufswahlvorbereitung}

Mädchen und Jungen schätzen ihre eigene Berufswahlvorbereitung unterschiedlich hoch ein. Dabei bewerten Jungen ihre Vorbereitung im Durchschnitt höher, als Mädchen dies tun. Dieser Befund bedeutet jedoch nicht zwangsläufig, dass Mädchen tatsächlich schlechter vorbereitet sind, er bestätigt vielmehr bisherige Beobachtungen in der Berufsorientierung und anderen, angrenzenden Bereichen der Bildungsforschung. Lazarides und Ittel (2012) zeigten in ihren Analysen zum mathematischen Selbstkonzept, dass sich Mädchen bei gleicher Leistung hinsichtlich ihrer mathematischen Begabung tendenziell schlechter einstuften als Jungen. Bezogen auf die Berufswahl betrachten Mädchen diese insgesamt kritischer (Fend, 1991) und prognostizieren für sich selbst einen geringeren Erfolg, als ihre männlichen Altersgenossen dies bei gleichen Leistungen und gleichem Selbstkonzept tun (Schuchart et al., 2016). Folglich sollten Mädchen in ihrer Sicherheit der eigenen Wahrnehmung gefördert werden (vgl. Cassie \& Chen, 2012; Kessels \& Hannover, 2004, 2007, 2008), um sie im Prozess der Berufswahl zu stärken.

\section{Zusammenhänge zwischen der Muttersprache und der empfundenen Berufs- wahlvorbereitung}

Auch im Zusammenspiel zwischen der Muttersprache und der Berufswahlvorbereitung sind Unterschiede dokumentiert. In allen Analysen zeigte sich, dass deutsche Muttersprachler*innen sich tendenziell besser vorbereitet fühlen als ihre anderssprachigen Peers. Darüber hinaus konnte für drei BO-Maßnahmen, nämlich das Praktikum, das Bewerbungstraining und die Jobmesse, jeweils eine signifikante Zweifachinteraktion mit der Muttersprache beobachtet werden. Daraus ergab sich, dass deutschsprachige Lernende unabhängig ihres Geschlechts und ihrer Schulleistungen von diesen Interventionen profitieren. Bezogen auf das Praxislernen können noch weiter ausdifferenzierte Aussagen getroffen werden. Von dieser Maßnahme profitieren Lernende mit deutscher Muttersprache, die im Vergleich zur übrigen Stichprobe nur schwache bis durchschnittliche Schulleistungen vorweisen. Sie schätzen ihre subjektive Berufswahlvorbereitung bei Teilnahme am Praxislernen vergleichsweise höher ein als Lernende im Regelunterricht. Ein weiterer interessanter Effekt konnte beobachtet werden: Obwohl das Praxislernen für schulmüde, abschlussgefährdete Jugendliche konzipiert ist, zeigt sich, dass auch 
überdurchschnittlich leistungsstarke Schüler*innen mit einer anderen Muttersprache als Deutsch einen signifikanten Nutzen aus der Maßnahmen ziehen. Eine mögliche Erklärung liegt in der Natur des Praxislernens selbst begründet. Granato (2013) stellte unter Bezugnahme auf die Imdorf-Studie (2010) zur sozialen Ungleichheit im Auswahlprozess von Betrieben fest, dass die Ursachen der geringeren Erfolge von Jugendlichen mit Migrationshintergrund bei der Ausbildungssuche eher in den Auswahlprozessen der Unternehmen, als in den Jugendlichen selbst verortet seien könnten. Durch das Praxislernen haben anderssprachige Jugendliche die Möglichkeit, sich innerhalb eines Betriebs über einen längeren Zeitraum abseits möglicher Vorurteile durch ihre individuelle Leistung zu beweisen und damit für einen Ausbildungsplatz zu empfehlen. Die von Granato (2013) aufgezeigten Nachteile Jugendlicher mit Migrationshintergrund werden so vermutlich zu einem gewissen $\mathrm{Maß}$ nivelliert.

\section{Gute Schulleistungen - ein Indikator für eine gute Berufswahlvorbereitung?}

Die Schulleistung stellt offensichtlich einen wichtigen Faktor in Hinblick auf die durch die Heranwachsenden wahrgenommene Berufswahlvorbereitung dar. Je besser der Notendurchschnitt der Lernenden in den Unterrichtsfächern Mathematik, Deutsch und Englisch war, desto positiver schätzten diese ihre Berufswahlvorbereitung ein. Diese Beobachtung reiht sich logisch an frühere empirische Befunde unter anderem von Stangl und Seifert (1986) zur Abhängigkeit der Berufswahlreife vom „Leistungs- und Bildungsniveau“ (S. 6) von Hauptschüler*innen an. Gleichzeitig kann jedoch angenommen werden, dass die Schulnoten eine allgemeine Verhaltenstendenz der Lernenden betreffs ihrer Leistungs- und Explorationsbereitschaft manifestieren. Super stellte in einem Interview mit Freeman (1993) dazu die Hypothese auf, dass die Berufswahlreife vor allem vom Berufswissen und der Bereitschaft berufliche Entscheidungen zu treffen abhängt. Die signifikante Interaktion zwischen der Schulleistung Jugendlicher und ihrer Teilnahme an einer Potentialanalyse lässt sich ähnlich interpretieren. Schüler*innen mit überdurchschnittlichen Leistungen ziehen einen signifikanten Nutzen aus dieser Intervention. Für Lernende mit durchschnittlichen oder schwachen Leistungen konnte kein signifikanter Effekt festgestellt werden. Daraus kann geschlossen werden, dass Lernende mit einer hohen Bereitschaft sich mit ihrer beruflichen Zukunft auseinander zu setzen aus der Potentialanalyse einen Nutzen ziehen, da für sie die Identifizierung ihrer Stärken und Fähigkeiten eine konkrete Unterstützung ihrer Fähigkeit zur Berufswahlentscheidung darstellt. Driesel-Lange und Kracke (2017) kommen hinsichtlich ihrer Untersuchungen der Potentialanalyse in Nordrhein-Westfalen zu dem gleichen Resultat, dass insbesondere Jugendliche mit stark ausgeprägter Berufswahlkompetenz von der Maßnahme profitieren. 


\section{Zusammenhänge zwischen BO-Maßnahmen und subjektiver Berufswahlvor- bereitung}

Die schulischen BO-Maßnahmen tragen offensichtlich zum subjektiven Stand der Berufswahlvorbereitung bei, jedoch sind ihre hier gemessenen Effekte sehr klein. Dieses Ergebnis ist jedoch konform mit entsprechenden Messungen von Ratschinski und Struck (2016). Weitere Studien zu BO-Maßnahmen (Cassie \& Chen, 2012; Jordan, Gessnitzer \& Kauffeld, 2016; Kuhnke, 2014; Schuchart et al., 2016) adressieren andere Schwerpunkte beziehungsweise andere BO-Maßnahmen, sodass ein direkter Vergleich der Ergebnisse nur eingeschränkt möglich ist. Schuchart et al. (2016) schlagen vor, dass gruppendynamische Effekte in der Gestaltung von BOMaßnahmen mindestens berücksichtigt und bestenfalls genutzt werden sollten. Denn ihren Untersuchungen zufolge hat beispielsweise die Studienabsicht von Mitschüler*innen einen positiven Einfluss auf die Studienabsicht der Einzelnen. Dementsprechend stellten Jordan et al. (2016) in ihrer Feldstudie an einer deutschen Sekundarschule signifikante positive Effekte eines zehnwöchigen Gruppencoachings auf die Karriereplanung, Selbstwirksamkeit in der Berufswahl und den Stand der Berufswahlentscheidung der Teilnehmenden im Vergleich zur Kontrollgruppe fest.

Bezogen auf den empfundenen Nutzen einzelner BO-Maßnahmen stellte Kuhnke (2014) in einer Studie mit Jugendlichen und jungen Erwachsenen in Unterstützungsmaßnahmen zum Übergang Schule-Beruf eine zwischen Subgruppen differenzierte Nutzenwahrnehmung fest: Mentoring-/Patenprogramme sowie Freizeit- und Erlebnispädagogik wurde tendenziell von männlichen beziehungsweise jüngeren Teilnehmenden und Teilnehmenden mit Migrationshintergrund als nützlicher für den „weiteren beruflichen Bildungsweg“ (Kuhnke, 2014, S. 1) eingestuft. Darüber hinaus empfanden Migrant*innen die Teilnahme an Bewerbungstrainings nützlicher als Jugendliche ohne Migrationshintergrund. Die Ergebnisse der hier vorgestellten Studie stehen dazu im Kontrast. Jugendliche mit deutscher Muttersprache schätzten ihre subjektiv empfundene Berufswahlvorbereitung signifikant höher ein, wenn sie an einem Bewerbungstraining teilgenommen hatten. Im Gegensatz dazu konnten keine signifikanten Effekte für Teilnehmende mit Migrationshintergrund festgestellt werden.

Die Teilnahme an einem Lebens- und Berufsorientierungskurs trägt, wie Cassie und Chen (2012) anhand ihrer Studie an einer kanadischen Sekundarschule zeigten, bei Mädchen und Jungen unterschiedlich zur Entwicklung von Teilaspekten der Berufswahlreife (vgl. Super et al., 1996) bei. Teilnehmende Mädchen erzielten im Vergleich zu ihrer Kontrollgruppe signifikant höhere Übereinstimmungswerte zwischen den von ihnen geäußerten und den für sie als passend getesteten Berufsinteressen. Bei Jungen verläuft der Abgleich zwischen Interessen und Berufswahl 
wiederum ähnlich, unabhängig einer Kursteilnahme (Cassie \& Chen, 2012). Allen Studien gleich ist die differenzierte Einschätzung und Annahme der Maßnahmen durch die verschiedenen Teilgruppen. Dies spricht wiederum für eine differenzierte Maßnahmenplanung.

\section{Grenzen der Studie}

Die dargestellten Untersuchungen beruhen auf einer Querschnittsdatenanalyse, also Daten eines Messzeitpunktes, sodass die Richtungen der dargestellten Zusammenhänge nicht abschließend geklärt werden können. So könnten sehr gute Schulleistungen ein Indikator sein, dass Jugendliche sich intensiv um ihre (berufliche) Zukunft kümmern, entsprechende Maßnahmen besuchen und sich dadurch besonders gut vorbereitet fühlen. Vice versa könnte auch eine subjektiv weit vorangeschrittene Berufswahlvorbereitung, im Sinne eines konkreten Ausbildungsoder Berufswunschs, dazu führen, dass die Lernenden eine zusätzliche Motivation für den Unterricht erfahren und sich daher auch schulisch mehr bemühen. Für die genaue Überprüfung der Wirkungsrichtungen wäre eine längsschnittliche Datenanalyse notwendig. Aufgrund der hier gemessenen, geringen Effektstärken der BO-Maßnahmen und ihrer Interaktionen muss neben der möglichen Schlussfolgerung tatsächlich geringer Wirkung auch die Möglichkeit in Betracht gezogen werden, dass die Effekte schwer messbar sind oder sich erst über einen längeren Zeitraum zeigen, sodass eine längsschnittliche Betrachtung - auch über den Schulabschluss hinaus - unternommen werden müsste.

Eine alternative Erklärung für die geringen Effektstärken der BO-Maßnahmen kann in zwei weiteren Fakten gefunden werden: Während der Erhebungen zeigte sich mehrfach, dass sich die Schüler*innen zum Teil nur schwer an bereits besuchte Maßnahmen erinnern konnten. So ist es denkbar, dass Jugendliche Maßnahmen, die ihre Vorbereitung gegebenenfalls maßgeblich unterstützt haben, nicht angaben und diese folglich nicht in den Analysen berücksichtigt wurden. Darüber hinaus gibt es in Berlin zurzeit keine einheitlich geltenden Durchführungsstandards und Nennungen für BO-Maßnahmen. Sich hinsichtlich der zeitlichen Dauer und des Durchführungsformats unterscheidende BO-Maßnahmen können also den gleichen Titel tragen. Die Teilnehmenden bezogen sich in ihren Antworten also möglicherweise auf unterschiedliche Maßnahmen. Um eine bessere Vergleichbarkeit zu erzielen, wäre eine Standardisierung der BO-Maßnahmen betreffs ihres zeitlichen Rahmens, der Methodik und der gewünschten (Lern-)Ziele daher wünschenswert.

\section{Implikationen für Forschung und Praxis der schulischen Berufsorientierung}

Die Ergebnisse zeigen klare Bedarfe einer individualisierten schulischen BO auf und liefern gleichzeitig erste Ansatzpunkte zur Umsetzung einer solchen. Nicht zuletzt 
dienen die Befunde einer weiteren Sensibilisierung in Hinblick auf die unterschiedlichen Effekte der Berufsorientierung auf Heranwachsende. Für weiterführende Handlungsempfehlungen müssen nun zusätzliche Aspekte betrachtet werden. Möglich scheint beispielsweise die Effekte der BO-Maßnahmen auf die Entwicklung von Berufswahlkompetenzen hin zu beleuchten, um eine noch feinmaschigere, zielgruppengerechte Differenzierung der Interventionen $\mathrm{zu}$ ermöglichen, die laut Kremer, Beutner und Zoyke (2012) eine wichtige Grundlage zur Individualisierung der Berufsorientierung darstellt.

Open Access Dieses Kapitel wird unter der Creative Commons Namensnennung 4.0 International Lizenz (http://creativecommons.org/licenses/by/4.0/deed.de) veröffentlicht, welche die Nutzung, Vervielfältigung, Bearbeitung, Verbreitung und Wiedergabe in jeglichem Medium und Format erlaubt, sofern Sie den/die ursprünglichen Autor(en) und die Quelle ordnungsgemäß nennen, einen Link zur Creative Commons Lizenz beifügen und angeben, ob Änderungen vorgenommen wurden.

Die in diesem Kapitel enthaltenen Bilder und sonstiges Drittmaterial unterliegen ebenfalls der genannten Creative Commons Lizenz, sofern sich aus der Abbildungslegende nichts anderes ergibt. Sofern das betreffende Material nicht unter der genannten Creative Commons Lizenz steht und die betreffende Handlung nicht nach gesetzlichen Vorschriften erlaubt ist, ist für die oben aufgeführten Weiterverwendungen des Materials die Einwilligung des jeweiligen Rechteinhabers einzuholen. 